\title{
LA RELIGION
}

Jaime Moreno G.

Abocarse al trato genérico del tema de "la Religín" im plica uno opcibn entre distintas posibilidades de método. De hecho, es posible enfrentar este problema desdo distintos ángulos:

a) Un enfoque comparativo, que procede por

- elaboración de "tipos" religiosos, abstraidos del tiempo y del espacio.

- comparación de sus mutuas semejanzas y diferencias.

- planteo de problemas a partir de la comparación hecha.

Ventaja de este metado es la adquisición de una flexibilidad de pensamiento. Quizbs lo mós importante que se logra es formula ción de nuevos problemas. De suyo, el metodo ilustra sin expli car ni probar nado.

b) El enfoque sociolbgico se preocupa de la función social de la religion. Trata de descubrir el ligamen estructural que une las redes religiosas con las restantes redes sociales, la función so cializadora e identificadora que realiza al interior de una comunidad.

c) Enfoque historico: Busca desentrañar las viciscitudes del hecho religioso. Asf, distingue lo nuevo de lo antiguo; descubre la supervivencio de lo antiguo balo formas nuevas; considera acaso el horizonte vital y el pensamiento que se ha ampliado o circunscrito.

Tanto el enfoque comparativo como el historico corren un grave riesgo al reconstruir tipos en base a datos aislados: la arbitroriedad acecha. Propio es el margen de error interpretativo con dicionado por presupuestos evolutivos (del tipo: "las formas más avanzadas son posteriores a las mas primitivas") y calificativos de los tipos logrados.

d) Si se enfoca la Religión desde el punto de vista psicológico, entonces habró que considerarla en lá vidá psiquica del indi viduo, descubriendo las relaciones entre la experiencia religioso y otras que le sean compatibles (vg. lo poesfa) tanto en los nive les de expresión como de contenido.

e) En el enfoque fenomenológico, el sujeto parte de lo que se mues tra de la Religión y a través de una progresión on su vivencia qulere llegar a la "comprensión" de la Religión, es decir, a la percepción del sentido de las relaciones que mantienen la organi- 
cidad de la estructura religiosa.

Un orden metódico y cientifico, deberlo entonces partir de las religiones o de las vivencias religiosas pora llegar, -si es posible-, a la Religibn. Nosotros seguiremos aqui un camino inver so: vamos a presentar una especie de "teorla general" de la religión, usando indistintamente los enfoques descritos. El tratamiento serb abstracto, procurando mostrar la forma religiosa, sin preocuparnos de los contenidos. Para eso estarán los capltulos siguientes.

\section{Procederemos en dos momentos:}

1. La Reilgión como reloción con lo Absoluto.

La experiencia y el símbolo.

2. Lo Absoluto como fundamento.

La función de la Religión.

3. Las actitudes religiosas.

1. La Religión como relación con lo Absoluto. La experiencia y el simbolo.

Los religiones, algunas de manera muy explfeita y otras de una forma un tanto mós implícita, tieneh una serie de componentes que llamoremos las instituciones religiosas.

En primer lugor, las religiones tienen ritos religiosos, tienen de una u otra manera doctrinas religiosas y como consecuencia de estas doctrinas, tienen exigencias éticas de comportamiento.

La pregunta es: EQue busca la Religión a traves de los rí tos que celebra? ¿Que es lo que pretende expresar a traves del apa rato doctrinal, conceptual que posee? ìA que responden sus exigen cias eticas?

De manera sumamente amplia y formal, dirlamos que lo que la Religib́n busca es al contacto y la relación con lo Absoluto. Cuando usamos la palabra "Absoluto" no le damos ningón contenido todavía: el contenido se lo dan las distintas religiones. Incluso intencionalmente evitamos emplear la palabra "Dios", por motivos que veremos inmediatamente.

Quizás para ver mós claro en este asunto vale la pena de- 
tenerse un poco a pensar en esta palabra "Dios", que clertamente es un concepto central deritro de la Religión y en los distintos conto nidos que se le, darón:

Hogamos un chequeo: EQQué significa la palabra Dios?

La respuesta espontónea es

- El Ser Supremo

- Lo Absoluto

Nosotros somos deudores: Nuestra palabra Dios no es ni siquiera una traduccibr, sino una trasliteración, es decir, una mera puesta en letras castellanas de la palabra originaria "Deus" Ia cual a su vez no es sino la trasliteración de la palabra griego "Theos". Y llegamos aquf, desde el punto de vista filológico, a una ralz. $Y$, zqué quiere decir "Theos"? Nos va a parecer extraño su significado. Si nos fijamos, la palabra "Theos" pertenece a la misma familia de la palabro "Theatron". Podrlamos hacer el repertorio de palabras que estón en la familia: Tenemos teatro, teorfa, teore ma; todas ellas pertenecen a la misma familia de la palabra Theos.

griega.

A la ralz de todo ésto parece haber una concepción muy

¿Que es el "teatro". Para el griego "theatron" es lo que se ve, lo que se contempla ahi en la escena. ¿Que es una "teo rfa"?. "Theorfa" no es lo que nosotros estamos acostumbrados a señalar como teorla, sino que teorfa es la contemplacion. "Theos" es una especie de adjetivo sustantivado que significa "lo más digno de ser contemplado". La polabra Dios significa, de alguna manera, lo mós digno de ser contemplado.

Esto revela en nuestro campo lingüistico, digamos en el Grea indogermana, una actitud bastante intelectual frente a Dios, qui z6s un tanto afectiva también, en el sentido de que cuando uno con templa la belleza, contempla la bondad, hace nacer el amor.

En cambio, si nos trasladamos a otras lenguas, por ejemplo, al mundo semitico donde estón nuestros antepasados judfos, hallamos que la forma mos radical de decir Dios es El. El es una ralz, que puede tomar bastantes formas como "il"; "UI". Notemos que "ul" es un nombre tecnico para decir "soldado", "fuerte". El, es "el fuerte", "el energetico", "el poderoso".

Desde luego, se puede hacer extrapolaciones quizbs hasta un tanto indebidas: En la medida en que la lengua refleja eso que 
los romónticos llamában "el genio de los pueblos" tendriamos concepciones de Dios absolutamente distintas en el mundo indoeuropeo $y$ en el mundo semitico.

En el mundo indoeuropeo, Dios es aquello que se contempla. La forma que adquiere lo Absoluto es la de lo digno de ser con templado. En combio, en el mundo semltico, Dios es lo Absoluto, pé ro en cuanto fuerzo absoluta, poder absoluto, energlo.

Por lo menos a nivel de la lengua, la concepción de Dios en el mundo indoeuropeo quedo en lo que se llamarla el extasis, el Extasis de la contemplación, la salida de si mismo, que se mara villa delante de, valga la redundancia, la "Maravilla contemplada".

El semita no. No se asombra delante de esta maravilla que sa contempla, sino delante de los hechos asombrosos de Dios, lo que es otra cosa.

En los primeros hay un matiz mós estático y uno mós dinámico, más activo en los segundos. No dirfamos si más concreta o menos concreto; mb́s productivo quizbs, mós cerca del pragmatismo. Por un lado un Dios "pragmbtico", por otro un Dios "tebrico": una concepción pragmbtica de lo Absoluto y una concepción teb́rico.

Retomemos lo dicho acerca de lo que hemos llamado la institucionalidad religiosa. La intencion que ponfamos en los ritos, en la doctrina y en la moral, tiene como punto de partida "la expe riencia".

Hemos dicho, a un nivel muy abstracto que la Religión busca el encuentro con lo Absoluto. El punto de partida y de llegado de la Religión tiene que ser ese encuentro con lo Absoluto, como to te encuentras con lo Absoluto. El encuentro es lo que se llama la experiencia. Por supuesto que aqui hay experiencias que son de distinto Indole, de distinto calibre, de distinto nivel. Y los caminos para esta experiencia de lo Absoluto son variados. Zuvien no ha leido a Pascal? Pascal sentía que esta experiencia de lo Abso luto era lo terrible, lo inmenso, lo enorme. Pascal ero un matemati co y un amante de los cielos y la vivencio de lo enorme del cosmos que se hace sentir, que se viene encima de nuestro pequeño mundo fue para $e l$ un camino de la experiencia de lo Absoluto. Es una experiencio de corte matembtico, pero no es la ónica.

Hay otra experiencia de lo Absoluto que vo o traves de lo poetico. Imaginamos que los pintores, los poetas, entienden por fectamente de que hablamos cuando decimos que se arremolinan los "de monios" propios de cada uno. Estos se apodoran del artista y lo po = nen frente a lo indecible. Ese es el momento en que se revela el hom bre literato que es capaz de decir lo indecible; el pintor que re = presenta lo irrepresentable y le da una forma. Ahl es, también, don de entra el hombre roligioso que as capaz de dorle un contenido a lá 
experiencio de lo Absoluto, a esto que es indecible, irrepresentable por definicion.

La experiencia puede ser de corte matemático, de corte poetico, puede ser la experiencia de corte social, por ejemplo, el horror de la guerra. Notemos que la experiencia de lo Absoluto puede ser no siempre una experiencia entusiasmante; hablóbamos de 10 aterrorizante que era la enormidad para Pascal. Podemos hablar de lo repelente, lo tremendo, del asco que producen por ejemplo las degeneraciones que trae una guerra consigo, o las que acarrea la mise ria profunda.

Hoy que destacar aque lo que llamariamos las experien cias límite: el sentir lo precario de la existencia, la necesidad de optar por un sentido de la vida y de la muerte.

A partir de estas experiencias brota la necesidad de buscar "Algo" que de sentido.

Franz Werfel fue un autor judfo, que escribi6 "La Canción de Bernardita". El puso en el frontispicio del libro un pe queño pensamiento: "Para quien ha hecho la experiencia, no es necesa ria ninguna explicación; y para quien no ha hecho la experiencia, no hay explicación posible". Creemos que para la cuestión de la expe riencia religiosa, esto es totalmente pertinente.

¿Y todo esto para quę Después de experimentar el no sentido de todo esto, cuando se ha sentido que se está como suspendido en el vacio, aparece lo Absoluto.

Por ser lo Absoluto se presenta con algunas características señaladas por los fenomenológos de la religión. Suelen hablar del "vértigo de lo Absoluto". Cuando se habla de vértigo, se habla de algo que atrae y que, al mismo tiempo, produce repulsión. Notémos lo bien: es una verdadera atracción y una verdadera repelencia.

Este "theos", este Dios, es atractivo y repelente, es terrible, es espantoso. Lo santo, lo numinoso, como se dice, son pa labras para expresar más o menos lo mismo: la atracción y el terror.

Recordemos las veladas de niño en el campo. All1, en el campo, una de las entretenciones favoritas era ( żlo es aún?) el contar cuentos de aparecidos, de "penaduras" y cosas por el estilo. ¿Que sucedla con nosotros cuando contaban esas historias? Estábamos al mismo tiempo, pendientes para no perder una palabra y al mismo tiempo con un miedo espantoso. He aqul un análogo del fenómeno ambivalente de la atracción y repulsión, tensión ambivalente desencado nada al entrar en el mundo de lo desconocido. Algo semejante ocurre en el reino de las experiencias del amor, que siempre deja "esa vieja herida que me duele tanto", como dijo el poeta. Alguien dijo 
por ahl que comenzar a amar es comenzar a sufrir, y ciertamente $e l$ amor-sufrimiento atrae y repele.

Este tipo de experiencias, la experiencia de lo Absolu to, por ser un encuentro que es muy personal, como lo es todo en.cuentro, es intransferible. Esto quiare decir que la experiencia de otro a uno no lo sirve; puede aceptarse, pero se acepta sobre la palabra de otro, no como vivencia propia.

experiencia.

¿Cuándo lo va a "saber" uno mismo? Cuando pase por la

Pensemos en el primer amor, en el primer beso. El adolescente quiere compartir lo experimentado con alguien. ácómo lo va a contar? Si el amigo ha pololeado y sabe de que se trata, entiende y el campo de referencio es claro. Si se quiere describir el sa bor del caviar a quien nunca lo ha comido żcómo contárselo? "Sé parece a las moras, pero tiene sabor a pescado" Imermelada de mora con gusto a pescadol... En cambio, cuando uno ya lo probs enton ces sabe. Pora quien hizo la experiencia no es necesaria ninguna explicación y para quien no la ha hecho, es dificil explicarselo.

Estas experiencias son intransferibles. Pero de todas

maneras la experiencia se vuelve objeto de reflexión. Hay como dos pasos en este proceso, primero en la experiencia misma y luego su comunicación. La niña que contaba su primer beso źqué es lo que es to haciendo? Tomando su experiencia y procurando convertirla en objeto, describirla para que el otro sepo cómo es.

¿Qué se hace cuando se da uno explicación? Se trata de crear un marco de referencia tal que la otra persona comprenda de que se esto hablando. Dado que la experiencia es intransferible, lo que se hace es convertirla en un objeto. Asi la experiencia es transferible en cuanto objetivada. ZComo se convierte la experiencia en objeto? A traves del lenguaje. En otras palabras, en forma muy global, a traves de símbolos.

Retomemos la experiencia religiosa: Ella se encuentra objetivada en símbolos religiosos de distinta Indole. All1 entran los ritos y las palabras, esas palabras "santas" que no pueden usar se de cualquier manera, esas palabras que son advertencia..... toda la simbología o red simbblica religiosa.

Al11 entran tambien las acciones simbólicas, en fin, todo lo que trata de alguna manera de objetivar la experiencia.

¿Que es un símbolo? Un símbolo cuando es verdaderamen te símbolo pretende decir lo indecible. Dirlamos que es exactamente como el cuadro que pinto el pintor, como la música que compuso el músico, como el poema que compuso el poeto, como el rito que creb 
el hombre religioso: En el mismo plano, con distintos recursos tratan de decir de alguna manera aquello que no se puede decir.

Quizós, el ejemplo de la música puede ser elocuente. Una persona que ha tenido una profunda experiencio estética, sensitiva, muy profunda, trata de volcarla y lo hace por el camino musical. ZEs que en la música estó lo experiencia? No, ese "demonio" mío, ese "de monio" musical que tengo dentro me ha empujado a dar esa forma a mi experiencio.

¿Y la simbología religioso? Asf como hay un "demonio" músical hoy tambien un genio religioso que conduce a los grandes fun dadores de rellgiones y los 1 leva a crear tales formos que de alguná manera objetivan la experiencia religiosa.

Hay que hacer notar bien que el símbolo no es la expe riencia, ni pretende describirla. El símbolo trata de decir lo inde cible y decirlo en cuanto indecible. Desde el momento que lo hiciera decible, simplemente lo volcarlo a un aparataje logico y conceptual en que lo experiencial desaparece.

El símbolo no es "intelectual", no es 16 gico, porque 10 intelectual y 16 gico es un todo coherente, decible, demostrable y manejable. El Símbolo, por el contrario, maneja a quien a $\ell 1$ se somete (pensár, por ejemplo en la patria, lo bandera, etc.)

El símbolo religioso por su condición de tal no puede ser reducido a un discurso, sino que es una especie de campo de encuentro en el cual podemos participar, compartir la experiencia religiosa.

Hay pues, que afirmor que la religión exige de suyo el desorrollo de un mundo simbolico.

La objetivación de la experiencia religioso es necesario mente simbolica; es distinto de lo $16 \mathrm{gico}$ y de lo racional, y quiere hablar de algo que está mós alla del hombre, fuera de $\epsilon 1$.

Ya hablamos del encuentro con lo Absoluto. Lo Absoluto no es reducible a lo relativo. Es este hombre, esta persona relativa quien quiere decir lo Absoluto, el cual por definición no puede ser encosillado. Quiere, pues, decir lo Absoluto y no puede. ¿Que hace? Crea símbolos.

El símbolo viene de la experiencia. Es su hijo legitimo. pero también es su padre: La simbología religiosa quiere ser aceptada pora que entonces cada uno pueda crear su propio experiencia religiosa.

Quien tuvo la experiencia religioso trata de objetivarla 
para que otros de alguna manera 1 leguen a comprender la. ¿̇Cuándo se logra la comprensión perfecta? Cuando a través del mundo simbolico se logra recrear una experiencia propia.

Notemos bien: las experiencias son intransferibles. Entonces, la experiencia religiosa de la que se origino determinado simbolo, motiva por su intermedio a la creación de la experiencia religiosa de quien en 61 se adentre. Pero la experiencia derivada del símbolo participado no seró igual a la original. Se acercarb a alla, puede que sea más intensa, de mayor o menor calidad: pero va a ser distinta. Por el simbolo se crean experiencios propias, no se reproducen experiencias ajenas. Al hacerlo "se comprende" de que se trata en la vivencia religiosa.

Lo que quisiéramos dejar claramente establecido es que la Réligión viene de la experiencia con lo Absoluto; que a partir de esa experiencio se crea un mundo simbblico. El mundo religioso es eminentemente un mundo simbolico, en el cual se representán cosas irrepresentables, en cuanto irrepresentables. Se dicen cosos indeci bles en cuanto inefables. Y ese mundo simbólico se ofrece abierto para que todo el que a $\$ 1$ se ocerque pueda crear uno experiencia pro pia.

Ahora bien: los simboloo estón sujetos a la entropía, su energía se desgasta: se formalizan, se institucionalizan, se ruti nizan y ya no son vivenciales. Se vuelven "cosas que hay que ha$\operatorname{cer}^{\prime \prime}$ y pierden justamente la característica de "símbolos que hay que experimentar". Cuando un símbolo se cosifica sucede algo semejante al que frente o un cuadro reacciona diciendo: "Si, es bonito. Esta bien. ¿Cuónto tiempo habró tomado pintar esto? ¿Cuónto costarb?"

Gran parte de las reformas religiosas -siempre necesa rias-, tienden a recargar los símbolos gastados con su ienergía nativa.

Una Gltima observación en este punto: Pensemos en el que ha objetivado su experiencia. Lo hizo y la ofrece. Ahora es necesario interpretarla. Es muy posible que un crltico literario descubra en la obra implicaciones o alcances que pasaron desapercibidas a su autor. Pensemos en Don Quijote de la Nancha, por ejemplo: Es muy posible que don Miguel de Cervantes y Saavedra no se haya dado cuenta muy bien de lo que estaba haciendo. A lo mejor fueron otros los que se dieron cuenta de su proyección. Eso no le quita el merito al autor: Quizós es el don y la maldición del artista el de ha cer cosas que ni el mismo sabe que son. 
2. Lo Absoluto como fundamento. La función de la Religión.

Al principio, dećanos que ibamos a tratar de la Religion como relación con lo Absoluto, de la experiencia, del símbolo y luego de lo Absoluto como fundamento. De esto Gltimo quisiáramos preocuparnos ahora.

Decíamos que la experiencia de lo Absoluto puede darso de mil y una maneras distintas; que cada uno tiene su camino con sus más $y$ sus menos.

Existe lo que los fenomenólogos llaman las experiencias Ímites, o experiencias radiccles, profundas, que están absolutamente en la raiz, como la muerte, por ej. La muerte es una experiencia limi te y la vamos a experimentar todos.

Somos frógiles. Cada uno de nosotros conioce sus grietas internas, sabe qué puntos calza; sabe cuáles son sus terrores y sus $1 f$ mites. La experiencia de saber los límites, de conocerse como ser 1 i = mitado lleva a la ralz. Por supuesto estén también las experiencias correlativas, de lo sublime, del goce de la plenitud de la vida. Son los dos tipos fundamentales, la experiencia del limite sea por la muer te sea por la de la plenitud de la vida. Ambas se tocan al final, sé vuelcan en la necesidad de ir más allá, sabiendo que en la ida al más allá, se entra al reino de lo terriblemente atractivo.

¿Qué es lo que sucede en las experiencias de lo Absoluto? Prácticamente como correlativo, se adquiere la percepción de la propia limitación.

La relación con lo Absoluto, la experiencia límite, puede generar dos tipos de preguntas: Uno pone en cuestion lo Absoluto, no tanto cuestionando su existencia, sino más bien interrogando sobre él: ¿Qué es? ¿̇ucede? ¿̇Cómo pasa? ¿Existe? ¿̇Es posible? Parece que no existe, porque si existiera, no deberían suceder muchas cosas..." Toda una serie de preguntas que se plantean sobre lo Absoluto.

El otro tipo derivado de la experiencia se da cuando fren te a lo Absoluto se pone el Yo en cuestisn. No 10 Absoluto; 10 Absolu= to no se discute, se experiment $\delta$, se sube que está: al acecho, amena zante, prometedor, en esa mezcla de aitrcción y repelencia. Es el Yo el cuestionado: "¿̀Quién soy? ¿̇Cómo sucedo? ¿Para quê?"

Al primer tipo de preguntas pueden darse dos clases de respuesta: Por un lado una afirmación de lo Absoluto. Lo Absoluto es el. fundamento de lo relativo y estó mós allo de lo relativo. $Y$, ì que es lo relativo que interesa en este caso? Nosotros, el hombre. $Y$, Equé es 10 Absoluto? Lo Absoluto es la fundamentación del hombre. reconocimiento de lo Absoluto quiere decir que el hombre no tiene fundamento y una explicación en sí mismo, sino que su fundamento, su 
explicación -y su sentido- esto fuera de $\ell 1$. Estó "mós all6". Entonces, cuando se busco el sentido de la vida, al aceptar la afirmación de 10 Absoluto, se afirma que el sentido de la vida no estó en la vida, sino que está fuera de ella; en ese Otro que es Absoluto, que es distinto, que repele tambiên, pero que explica y fundamenta la existencia.

La otra clase de respuesta puede ser la negación de 10 Absoluto: No existe. Entonces, se ofirma que el fundamento de 10 re lativo est 6 en lo relativo mismo, que el fundamento del hombre est $\bar{b}$ en el hombre $y$ que el sentido del hombre es el hombre.

En la primera respuesta se decía: el hombre se explica por el Otro; en la segunda, que el hombre se explica por el hom bre. El fundamento del hombre estó en el Otro; el fundamento del hombre estó en sI mismo. El fundamento del hombre estó mós al16; el fundamento del hombre está más acá, adentro. El sentido y la interpretación del hombre lo da el hombre mismo: lo que $\varepsilon 1$ quiera ser.

O por Oltimo, la negación de lo Absoluto puede desembocar en que simplemente no hay sentido, no hay un significado, no hay un fundamento. Hay eso que se llamaba el bruto vivir, el simple existir, el estar. $Y$, épor que? Pues, porque estoy, porque s1. Se diró que la pregunta sobre el sentido, no tiene sentido.

Si nos fijamos, la cuestión fundamental es la siguiente: Acaso el hombre tiene sentido y fundamento para su existencia. Eso es lo primero. Y lo segundo dónde estb́ ese sentido y dónde estó ese fundamento.

Por aquí ya entramos a una cuestión paradb/ica: las religiones de lo relativo. Quien dice que la explicación del hombre estó en el hombre, por olltimo tendró que construir y buscar la expli cación del hombre en el hombre. Es decir, que el hombre es lo $A b=$ soluto. De ahi se seguirs uno interpretación volcada hacia adentro del hombre, una religión del hombre, de lo relativo considerado como Absoluto.

Parece que, desde este punto de visto, podemos hablar tranquilamente de religiones ateas. Se busca el sentido en prescin dencia de Dios. La existencia del hombre, se funda en una religión de la humanidad, religión del hombre.

Aqul quisiera enhebrar un paréntesis: Desde el punto de vista religioso judeo-cristiono, cuondo el hombre se convierte en lo Absoluto, en la explicación, entonces el mismo o sus creaciones se convierten en Dios. Estamos en lo que se llama la fabricación de Idolo: La evolución, el proletariado, la raza, la potria, la ciencia, la técnica, cuanto es creado por el hombre se puede transformar even tualmente en $\{$ dolo. 
Hoy entonces dos opciones religiosas posibles: Lo "religión de Dios" y la "religión del hombre", que visto desde el ju deo-cristianisino, desemboco en lo "religion del idolo".

De todas maneras, en esta relación con lo Absoluto 10 cantral es la experiencia. Pero no de la experiencia por la expe:riencia, sino por lo búsqueda del sentido, del fundamento del ser y dol quehacer del hombre.

Es el momento de preocuparnos de la función de la relí gión en el entramado cultural. ¿Qué "hoce" la religión en la culturo?

Partamos diciendo que el concepto de relación es 10 fundamental para entender la cultura. Diriamos que lo cultura es un modo global de relacionarse. Cono se relaciona una persona con otra, un grupo con otro; la forma de relacionarse uno con la comu nidad y con las cosas. Todo ese entramado de relaciones es lo que forma la cultura. No entraremos chora en los problemas de la cultu ra, cosa que nos llevaría un tanto lejos, simplemente deseamos po ner de relieve su aspecto relacional.

¿y que sucede cuando el hombre se relaciona consigo mismo, con la comunidad, con las cosas? Sucede que el hombre comien za a secretar una serie de lo que podrlamos llamar redes relaciona les. Distintas redes, y estos redes en conjunto, como una gran telaraña es lo que llamamos cultura.

Veamos algunos formas de estas redes, para considerar luego dónde y cómo se ubico la religión.

el lenguaje? 1 . Una primera red cultural es el lerguaje. tQue hace de su mundo cotidiano y las ileva a otro plano, al simb6lico. Por ejemplo, alguien ve a una goviota; el lenguaje le permite apoderarse de la gaviota y llevarla a otro plano que es de tal naturaleza que a partir del momento en que se logrb evacuar una relación lingǘstica formando la palabra gaviota, ya rio es necesaria la gaviota. De hecho aqul, en nivel lingüístico se peses la gaviota sin gaviota. Basta con la palobra. Y ésto permite taclo unc serie de juegos. Con el len guaje se sacan las cosas de su mundo cotidiano. Entonces es posiblēe un momento en el cual "las piedras" pueden ser "plumas" y "las plumas" pueden ser "piedras", no en el nivel de lo cotidaneidad de las cosas, pero si en el plono linguśstico. Y Esto no es simplemente un juego, sino que es una cosc muy seria. Hoy un plano en que verdadac. ra y realmente, las niñas pueden ser rosas y las rosas pueden ser ni ñas, donde ya la rosa dejó de ser rosa y la niña dejó de ser niña, y se ha creado una tercera realidad que ya no es rosa ni niña, sino que es una realidad lingülstica rosa-niña o niña-rosa: Es la metómfora. Es el plano en que "la brillante redoma llena de lagrimas", 
de Neruda, no es propiamente ni cebolla ni redoma, sino otra realidad: la redama ya no redoma, está lleno, de lágrimas; pero no es cebolla por que es redoma $y$ no es redoma porque es cebolla.

Una vez creadas las palabras y el lenguaje; ahora se pue de crear un mundo entero, un mundo que no existe en lo cotidiano, pero no por eso tiene existencia menos real: la existencia simbolica.

Estamos tocando un problema profundo, aunque pueda parecer un tanto pedante:

No podemos reducir la realidad simplemente a lo cotidiono, porque la realidad lingǘstica es tan real como lo cotidiano y quizás mós. Un ejemplo: Don Quijote de la Muncha, del cual ya hablamos des real o no? Evidentemente no es real en lo cotidiano, pero tiene su realidad lingüistica. Pongamos por un lado multitud de personos muy reales del mundo cotidiano, con todos sus valores y don Qui jote real "solamente" en el mundo lingüsstico, sin noda de cotidiano, realidad absolutamente simbolica. Resulta obvio que la radlidad 1 ingülstica es una cosa bastante serla, tanto o más que muchas anodinas realidades cotidianas.

EPor que la literatura es una cosa tan serias zPor que a veces se le tiene miedo a la literatura? $Y$, żpor qué otras veces se lucha por la literatura? Es "solamente" palabras, nada más que pa labras. Nada menos que palabras.

$Y$ áque es lo que hace la palabra? Crea mundos posibles. El lenguaje esta continuamente creando utopias, mundos posibles, que, no son simplemente la realidad cotidiana. Los libros estón diciendo que no necesariamente el mundo de lo dado debe ser como es.

2. Otra red cultural es la ciencia. La ciencio es también un mundo simbblico en el cual también las cosas son sacadas de su rea1 idad cotidiana $y$ convertidas en simbolos, en simbolos humanos. El espacio del geómetra, la "naturaleza" del físico, etc., no es el espacio ni la noturaleza del espontóneo experimentar cotidiano.

Las ciencias convierten el mundo en simbolos, y hacen exactamente lo mismo, aunque en otro plano, que la literatura; juega con los signos. Pensemos en el algebra, en la fisica, en la químico y on sus juegos combinatorios sustitutivos, etc. Alguien jugando por ahi descubris que energía es igual a $\mathrm{mc}^{2}$. Los signos llegaron a ser uno formula. Y esa formula como la literatura, es tambien seria.

El mundo simbrlico de las ciencios parte del mundo cotidiano, o mejor dicho, toma el mundo cotidiano, lo convierte en simbolos y despues vuelve al mundo cotidiano. La ciencia y todo su aporato simbslico, cientifico, tiene algo de lúdico, de adivinanza; pero cuan do vuelve al mundo cotidiano ya no lo deja como antes. El ingeniero, 
el artista, el calculista, cuando trabaja en su especialidad, tienen todo el cemento, todo el fierro pora curistruir un edificio, tienen todo un mundo posible. Y lo tiene todo ohs, en el número, en el croquis que yace en su tablero de dibujo.

3. Una tercera red cultural es la del derecho y la poli-

Aqui se diseñan las utoplas de las relaciones entre los hombres, las relaciones y los juegos de poder, de la autoridad, las relaciones entre los grupos sociales. Todo Esto esta puesto en un mundo simbólico, en un mundo discursivo. Como hay un modelo lingüistico, como hay un modelo lingǘstico de la realidad, hay tambien un modelo polftico que es posible y que luego se implementa.

El arte político -nuevamente 10 mismo- consiste en to mór la realidad cotidiana del mundo cotidiano, convirtiéndola en esto otra realidad que ya no lo es.

Todas estas redes (lingüistica, politica, artística) son las que formán la cultura. Sucede que no se pueden tocar una sin que se resiento todo el conjunto.

4. Llegamos finalmente a la red que ahora nos preocupa: También en forma simbólica, la red religiosa quiere dar sentido y fundamento al ser y quehacer humano.

André Jolles, en su libro "Las Formas Simples" dice que el hombre tiene tres actividades bósicas: es agricultor, artesano y sacerdote. A veces las tres juntas, a veces aparte.

El llama agricultor al ordenador, que ordena la tierra, quita las malezas, limpia, traza los surcos, distribuye las semillas, regula la secuencia del agua. Las cosas quedan como eran antes de la obra del agricultor. Lo que hizo fue ordenarlas. Desde este punto de vista toda actividad ordenadora es obra propia del agricultor. El agente del trafico ordena las cosas para que marchen mejor, pero din cambiarlas: $\in$ l auto quedo como auto; la calle, calle, etc. Gran parte, -si no la totalidad-, del trabajo burocrótico es, desde el punto de vista de Jolles, agrícola: Ordena.

La segunda actividad es la del artesano. ¿Qué hace el ar tesano? El artesano toma las cosas y las transforma en otras cosas: toma la realidad y la cambia.

Hay educadores que pueden ser "agricultores", si lo único que les interesa es que el niño sea ordenado. Pero el trabajo del educador es un trabajo "ortesanal". El educando tiene que cambiar. 
La tercera, es la actividad del sacerdote. El es quien dice que sentido tiene el trabajo del agricultor $y \in I$ del artesano. No hace nada, no hace cosas: no ordena ni transforma, sino que quie ro dar sentido a lo que se hace. Surgen especialistas en la búsqué da del sentido. Por ejemplo, los filosofos, eventualmente, los cientificos: El filbsofo hace labor sacerdotal. En cambio, la obra del tecnico es artesanal, desde el punto de vista de la tipologla señalada.

La religión, entonces, tiene como función buscar y empu jar hacia el sentido. La religión no quiere decir por que suceden las cosas, a nivel causal. Eso lo dirón, por elemplo, las ciencias, la sociología, la medicina. La religión quiere decir qué sentido tienen las cosas. Un ejemplo: zQuién dice por qué y cómo se gesta y nace un niño? El ginecologo, el embri6logo, el ingeniaro de la bio genética: ellos dicen el por que y cómo son las cosas. Pero hay otra pregunta que $\mathrm{ni}$ el ginecólogo, ni el ingeniero bioquímico puede contestar. Tiene que contestarla uno solo: ¿Que significa un hijo? żCubl es el sentido del hijo? Una mań puede ser absolutamente ignorante en las causas de las cosas; pero puede tener una sabidurla enorme en cuanto al sentido de las cosas. Puede, desde el pun to de vista de Jolles, ejercer perfectamente la función sacerdotal: sabe cubl es el sentido del hijo.

Declamos que todas estas redes, lingülstica, cientifi ca, polftica, artística y religioso, forman lo que llamamos el entramado cultural. Declanos, ademos, que estas redes están tan bien estructuradas, que no se puede tocar una sin que se comprometa todo el conjunto. Entonces, resulta que la religión tiene su función je rarquizada según el lugar y la trabazb́n que le corresponde dentro del conjunto cultural.

La religión no tiene un "status" asegurado en el conjun to: En cierto momento la religión pudo ser el punto de referencia de todas las demás redes, pero eso no es histbricamente necesario. Otras redes crecieron y se colocaron en su lugar.

Cuando hablamos de una "cultura religiosa", entendemos que la red religiosa es la que comanda: Alll, por Ultimo, las cuestiones cientificas se resuelven por un dictamen religioso, las cues tiones politicas por un dictamen religioso, la cuestión ortistica por un dictamen religioso, hasta el uso de las palabras.

¿Que pasa cuando ésto cambia y crece, por ejemplo, la ciencia? Todo el aparato debe readecuarse. Resulta claro constatar, pues, que se da una funcionalidad entre las distintas redes.

En nuestro mundo uno de los problemas que ha estado 61timamente sobre el tapete ha sido el aluste de la red religiosa en el sistena cultural. EQue tiene que decir la religión a la red cientifica, por ejemplo, a la ciencia económica? La religib́n esto 
dentro de la estructuración cultural, y el conjunto relacional de termina el papel que juega. Sobre todo va a ser muy interesante la relación que se dé entre religión, polftica y ciencia.

Por otra parte, hay que decir que las culturas están en evolución, que cambian. El equilibrio entre las redes culturales no es firme y estable, sino que esto en continuo movimiento. Las redes van tejiendose en la urdimbre cultural, desarrollándose una especie de equilibrio sanamente inestable. En el momento en que el equilibrio se estabilizara, si estuviera todo equilibrado, entonces se acabarla lo vida. El conflicto es la condición ineludible para lo creatividad. Si no hay conflicto es imposible que haya creación.

¿Qué estó sucediendo hoy con la religión dentro de la cultura? No nos atrevemos a hacer un diagnóstico, cuendo mós, vamos a emborronar algunas observaciones.

Por un lado, consideraremos la función socializadora de la religión. ¿Que quiere decir función socializadora? Quiere decir que un grupo social mb́s o menos solidificado y armado debidamen te, socializa, es decir, incorpora a los individuos al modo de ser del grupo. Veamos. Cuando en nuestras casas se corrige a los niños se hace una labor socializadora: el niño debe aprender que, en el grupo donde debo moverse no se mete la mano en el plato, por ejemplo.

Cada grupo tiene un lenguaje, un modo de hablar más o menos propio. Va a tener una concepción del arte mb́s o menos propia, va a tener modelos polfticos mas o menos propios, va a tener una mentalidad cientifica y un pensamiento religioso mós o menos propio. Todo eso, que forma la cultura, identifica al grupo. El gru po socializa cuando logra que los individuos se adapten a los modos culturales identificadores del grupo.

Elemento privilegiado de socialización es el lenguaje. Nosotros no nacemos sabiendo hablar; aprendemos a hablar, es decir, aprendemos a comunicarnos dentro de un grupo. Nos socializamos, por que detrós del lenguaje estó toda la herencia cultural. Detrás del niño que aprende a decir "mama", "papa", estó Cervantes; alguna vez tendró que encontrar a Don Quijote de la Mancha, o al Mlo Cid. 
¿Y la religión? La religión también es socializadora, es decir, integra gente a un grupo social. Desde este punto de vista, se puede hablar de una cultura cristiona en la medida en que hay un gru po por el cual es eristianismo es mús o menos un marco de referencia. Sobre todo cuando hay coyunturas en las cuales, por ejemplo, la religión se pone al servicio de modelos polfticos, de modelos cientifi cos, entonces su labor socializadora es mucho más sentida.

Por otra parte, ld religión puede funcionar como identifi cadora, justamente porque socializa: Identifica al grupo de "los judíos", al grupo de "los cristianos", al grupo de "los catblicos", al grupo de "los protestantes". Identífica, y los que estón en el grupo se sienten "yo cotólico", "yo judfo", "yo cristiono". La religión es, pues, identificedora.

En nuestro mundo se plantea el problema de hasta dónde es relevante esta función identificadora y socializadora de la religión. Escuchamos a veces decir a alquieri: "En aquellos tiempos en que todos Éramos católicos". "En esa Epoca en que quien no iba a misa los días domingos era señalado con el dedo". Como la práctica religiosa era identificadora en la sociedad también recibia la sanción social, la sociedad sancionaba. Ciertamente esto se ha aflojado.

Consideremos lo que sucede con la religión cristiana y si se quiere con lo religión católica. A los católicos, cuando llega la hora de la verdad żqué los identifica mejor? éla religión o los intereses? Cuando se habla de intereses no se habla de algo malo. Pueden ser intereses políticos, intereses econmomicos, intereses cultura les, etc. Eso no quiere decir que sean cosas malas, pero cuando $11 e=$ ga la hora de la verdad, ¿́qué es 10 que en Chile identifica més? católico "de izquierda" con un católico "de derecha"? ¿Un catblico "de izquierda" con un socialista o un comunista ateo? EUn catblico de derecha con un ateo "de derecha"? ¿Dónde hay mejor inteligencia?

Parece que la función socializadora e identificadora de la religión es débil. Sobre ésto no disponemos tanto de datos, cuanto de sugerencias. En los Estados Unicios se ha investigado el "Sta blishment" norteamericano "WHASP", es decir, el modelo tipo "White", "Anglosaxon", "Protestant" (blanco, anglosajón y protestante). La elección de Kennedy fue síntoma de que la "P" del WHASP estaba en vias de debilitamiento. Los socílogos de EE.UU. se preguntan źquién se siente más identificado con quién दै żUn negro catb́lico con un negro protestante 0 un negro catslico con un blanco protestante 0 con un blanco cotblico? Hay otros factores que identifican mós. ¿Quét identifica mbs, un italiano catblico con un escoces protestante, o con un holandés protestante? Uno es protestante, el otro católico, ambos son "White", pero estón fuera de "stablishment". 
Quizás valdría lo peno hacer la tipificación del "stai-: blishment" en nuestra sociedad, e interrogarse acaso la religiśn identifica y si acaso es deseable que la religión cumpla esa función.

En este momento teremos uno guerra entre católicos y pro testantes en Irlanda, pero la sensación fuerte es que esa guerra no es en realidad una guerra entre catblicos y protestontes; es una gue rra de otro tipo en la cual la religión es un elemento identifico dor, una especie de bandera.

3. Al principio hablabamos de que la religión parte de la experiencia, que la necesidad de objetivarla conduce a la creación de símbolos. Estos símbolos, paulatinamente, van adquiriendo una institucionalización y un reconocimiento. La religión se oficializa.

Cuando decimos "se oficlaliza" no significamos algo asf como que la Constitución Politica del Estado establezca determinada religión como oficial. Significamos que recibe una sanción social in dependiente de las anciones juridicas. Por ejemplo, en el Norte han estado socialmente institucionalizados los bailes chinos. Son una institución socialmente sancionada sin articulo legal alguno que lo establezca. Es la sociedad quien los ha odoptado y sancionado. Sa bemos por la historio que los bailes chinos fueron prohibidos varias veces, pero el cuerpo social se sentia mucho mis identificado con ellos que con cualquier decreto contrario.

Hablamos aqui de Religien oficial en este sentido, cuando la sociedad institucionaliza, oficioliza los ritos, las doctri nas, las conductas eticas de que hablabamos. Se hace a veces con leyes, otras sin ellas; la sanción escrita puede existir o no.

Por aqui se crea un probleme: En doctrina, en rito, en etica, hay una sanción social que no necesurianente coincide con la oficiel iegalizcida.

Ante la religión oficializado en la formo que declomos puede existir una actitud interralizoda. Por elemplo, la del cató lico practicante que pertenece a una "comunided de base" a un "movi miento", que va a misa todos les domingos, que cumple con todo lo es tablecido en forma internalizada, no sôlo porque esté mandado sinodesde adentro, aceptando todo. Los saciblogos dicen que esta actitud internalizada frecuentemente cónlleva una especie de mala conciencia. Consiste en que se dice "si" a todo, pero siempre hay algo de "mi" religión, que no encaja bien con el conjunto de la vida: Personas, decisiones que no cuadran. En la actitud internalizada siempre que dan aristas que molestan, pero se aceptan y hay uno adhesib́n global. 
La segunda actitud es la que se llama actitud retbrica. Si la religión oficial es católica, el retórico declara que "por su puesto, yo soy católico, apostólico, romano, pero a mi manera". "Bueno, yo soy católico pero no voy a misa, no ccmulgo, pero soy ca tólico", "Yo soy católico, pero no acepto la doctrina social ni lä regulación de los negocios ni de otras cuestiones. Esto se rige por otras reglos. Poro, yo soy católico".

En la actitud retórica, la adhesión a la roligión oficial es discursiva, de discurso.

Donde se adopto eso actitud un tento confusa hay consecuencias claras: la religión en lo religioso, lo econb́mico en lo económico, lo poiltico en lo político, lo cientifico en lo científi co, etc. Cuando toco funcionar en lo religioso se funciono como re ligioso, pero en el momento siguiente político, científico o económico yo no funciona la religión.

Esta es lo que los sociblogos 1 laman la actitud retórica, que no súlo se da en lo religioso sino en muchos otros ámbitos.

Una tercera actitud es la critica. Aqui se plantean los problemas y se tiene la lucidez suficiente para ver que la religión se da en un entramado cultural y que dicho entramado cultural estó en movimiento, que la religión no puede quedarse atrós, no porque haya que defender la religión sino porque la función religiosa es ineludible y debe ser cumplida. Entonces, se examinan donde ella va quedando atrás, donde va hacia adelarte su funcicnalidad y dis funcionalidad. Se posee una actitud critica ante la religibn.

Hemos procurado trator la Religión desde un punto de vista generico, formol. Las referencias a religiones concretas han querido sólo ilustrar lo genérico.

En los estudios siguientes se podrón encontrar los "contenidos" distintos que ha recibido y recibe esta "forma simbolica cultural". 